\title{
Ambientes educativos escolares: Una investigación sobre la propensión a aprender en jardines infantiles chilenos*
}

\author{
School educational environments: An investigation about the propensity \\ to learn in Chilean kindergartens \\ Ambientes educativos escolares: Uma investigação sobre a tendência \\ a aprender em jardins infantis chilenos
}

\begin{abstract}
Alberto Moreno Doña, ${ }^{a}$ Silvia López de Maturana Luna ${ }^{b}$
${ }^{a}$ Universidad de Valparaíso, Facultad de Medicina, Carrera de Educación Parvularia, Angamos 680, Reñaca, Viña del Mar, Chile. Teléfono: (56) 32 2603822. Correo electrónico: alberto.moreno@uv.cl

${ }^{\mathrm{b}}$ Universidad de La Serena, La Serena, Chile.

Teléfono: (56) 51 2204478. Correo electrónico: silvialml@gmail.com
\end{abstract}

\begin{abstract}
RESUMEN
Estudio Cualitativo centrado en el conocimiento y descripción de los ambientes que propician o entorpecen la propensión a aprender de los niños de 4 a 7 años a partir de la propensión a aprender que manifiestan. Utilizamos la observación etnográfica en Jardines Infantiles municipalizados en las ciudades de La Serena, Coquimbo y Valdivia. Los resultados muestran cómo los niños construyen sus aprendizajes de forma genuina, explorando y manifestando satisfacción por sus logros, aun cuando las educadoras construyen prácticas que invisibilizan la complejidad del pensamiento infantil.
\end{abstract}

Palabras clave: propensión a aprender, modificabilidad cognitiva, ambientes activos modificantes, ambientes pasivos aceptantes.

\section{ABSTRACT}

Qualitative study focused on the knowledge and description of environments that contribute or not to encourage the natural propensity to learn in children. Ethnographical observation in municipal kindergartens from La Serena, Coquimbo and Valdivia was used. Results show how children build their learning in a genuine way, exploring and showing themselves satisfied with their accomplishments, even though educators build practices that ignore the complexity of their thinking.

Key words: propensity to learn, cognitive modifiability, active-modificational orientation, passive-acceptant approach.

\section{RESUMO}

Estudo qualitativo focado no conhecimento e na descrição de ambientes que propiciam ou dificultam a tendência a aprender das crianças de 4 a 7 anos, a partir da tendência manifestada por elas. Utiliza-se a observação etnográfica em Jardins Infantis municipalizados nas cidades de La Serena, Coquimbo e Valdivia. Resultados mostram como as crianças constroem suas aprendizagens de maneira genuína, explorando e manifestando satisfação por suas realizações, enquanto as educadoras constroem práticas que invisibilizam a complexidade do pensamento infantil.

Palavras chave: tendência a aprender, modificabilidade cognitiva, ambientes ativos modificantes, ambientes passivos aceitantes.

Este artículo se realiza en el contexto del Proyecto de Investigación Fondecyt No 1110577, titulado "Asombros Educativos Infantiles y Propensión a Aprender" (2011-2014). 


\section{INTRODUCCIÓN}

En este artículo presentamos los resultados del proyecto de investigación Fondecyt $\mathrm{N}^{\mathrm{o}}$ 1110577, titulado "Asombros educativos infantiles y propensión a aprender". Nos planteamos, entre otros aspectos, que las prácticas culturales de la escuela inhiben, incluso mutilan, la propensión a aprender del ser humano. Si bien, este es un tema recurrente en los análisis críticos sobre la escuela, quisimos entregar antecedentes concretos de lo observado diariamente en las aulas de los niños desde los 4 a los 7 años. Este seguimiento exhaustivo nos permite señalar tendencias pedagógicas que muestran una escuela que desde los primeros años tiende a hacer desaparecer la genialidad connatural del ser humano.

Hemos investigado a niños, desde los 4 años de edad, que asisten al pre kínder durante el año 2011 y kínder en el 2012, a quienes hemos acompañado diacrónicamente en su desarrollo, observándolos en primero básico en el año 2013 y en segundo básico en el 2014. Los grupos son intencionados y los constituyen 16 niños/as distribuidos en jardines infantiles de escuelas de La Serena, Coquimbo y Valdivia.

Nuestros planteamientos se basan en la convicción de que los niños "naturalmente" propenden a aprender dentro de la cultura en la que conviven con los otros significativos, ese principio acompaña nuestras reflexiones y análisis sobre los procesos educativos que se dan dentro y fuera de las aulas. En este caso, nos referiremos a lo que ocurre en la educación formal donde se espera, y supone, que dicha propensión se promueva, enriquezca y actualice. De tal manera que esa propensión natural podría anquilosarse si no encuentra "tierra fértil" en la cultura escolar formal.

La propensión a aprender se entiende desde la enorme potencialidad de desarrollo que cada ser humano trae al momento de nacer, la que necesita de la intencionalidad de un adulto mediador que organice experiencias de aprendizaje óptimas para actualizarla y para que la genialidad de los niños no se anquilose (Calvo, 2012; Moreno, 2006; Moreno \& Calvo, 2010).

La genialidad en los primeros años es indiscutible y se observa en las preguntas, respuestas y reflexiones que así lo demuestran:

a veces me pasa eso, las palabras se confunden, se van para arriba, se dan muchas vueltas, hacen círculos en el aire y luego vuelven y cuando las palabras están arriba y mi mamá me habla, ahí se enredan sus palabras de color rosa con las mías que son de color verde ..., sí, porque mis palabras son verdes y las de ella son rosa ¿tú sabías eso? (Registro de campo).

Vicente, a sus cuatro años, da cuenta de una gran complejidad de razonamiento, característico de la mayoría de los niños de esa edad cuando viven experiencias de aprendizaje en ambientes enriquecedores. Nos preguntamos: ¿qué reacción tiene el adulto ante esa reflexión?; ¿le presta atención?; ¿ayuda a seguir reflexionando?; ¿lo lleva por otros derroteros para que su fantasía emerja con mayor potencia?; ¿establece relaciones o sucede lo contrario?; ¿lo calla y le ordena que vuelva a su tarea?

Desde sus primeros años, el ser humano necesita de un adulto que lo guíe, aspecto crucial en su formación. Es en la escuela donde esta intencionalidad se manifiesta con mayor fuerza favoreciendo o desfavoreciendo el natural cauce creativo. El principal soporte

\footnotetext{
Con este registro dimos inicio al proyecto de investigación. Nuestra principal intención era comenzar mostrando la genialidad infantil.
} 
educativo formal en los seis primeros años de vida son las educadoras de párvulos y luego los profesores, de quienes dependerá enriquecer o entorpecer la propensión a aprender ${ }^{2}$ característica de los/as niños/as de esas edades.

El foco de esta investigación no está puesto en el contenido a aprender, sino en las relaciones generadas entre los niños, entre estos y los adultos y también entre las relaciones con su entorno para que el aprendizaje sea significativo y los contenidos tengan sentido.

En la investigación hipotetizamos que la calidad de las relaciones en los primeros años es similar en cualquier tipo de contextos socioeconómico-culturales, porque los niños participan más activamente en la cotidianeidad de la educación informal. Sin embargo, a los dos años de investigación pudimos señalar que, si bien a esa edad las diferencias socio económico-culturales son menos notorias entre los mismos niños, estas se van marcando fuertemente y se comienza una artificial separación entre los que pueden y no pueden aprender, aun cuando todos tienen las mismas capacidades, aunque no las mismas oportunidades.

La escuela fomenta, desde los primeros años, la competitividad y la diferencia entre los que saben y no saben. Eso lleva a la exclusión y automarginación de los niños con dificultades para aprender, no obstante la institución escolar debiera ser un lugar donde el alumnado se sienta contento, motivado y con ganas de aprender.

Durante los primeros años de vida se construye el andamiaje (Wood et al., 1976) necesario para los aprendizajes posteriores. Para eso la escuela necesita crear ambientes activo modificantes donde a través de experiencias de aprendizaje significativas el profesorado pueda diseñar estrategias que partan desde los intereses de los niños, escuchar y poner atención a sus conversaciones y sacar provecho para que el aprendizaje sea trascendente (Calvo, 2004; Feuerstein, 1991). Se trata de transitar desde la curiosidad espontánea a la epistemológica y de posibilitar genuinamente que los niños y las niñas aprendan a situarse en su contexto social y cultural.

\section{MARCO TEÓRICO-REFERENCIAL}

\subsection{EDUCACIÓN INFORMAL Y PROCESOS DE AUTOORGANIZACIÓN}

En la investigación educacional la educación informal aparece relegada por la primacía de la educación formal y la no formal. La educación informal se entiende como el proceso de socialización que los niños vivencian al exterior de la escuela, o aquellos aprendizajes producidos en la escuela a partir del llamado currículo oculto (Calvo, 2012; Moreno, 2006).

Se posterga, por ejemplo, el estudio de los factores que causan el aprendizaje en los procesos informales, como el aprendizaje de la lengua materna, la adquisición de la locomoción, los valores y la socialización primaria.

Esto lleva a cuestionarse la separación existente entre el proceso de educación informal y el formal educativo escolar. La transmisión y re-creación de los principios de enseñanza y de aprendizaje de la educación informal a la escuela podría generar grandes cambios en las instituciones educativas formales, lo que podría contribuir a aminorar los malos resultados

Entendemos la propensión a aprender como la capacidad biológica e innata del ser humano para construir conocimiento estableciendo relaciones entre la experiencia vivida y las experiencias y desafíos nuevos que aparecen en la cotidianeidad de esos sujetos. 
académicos de los alumnos que poco y nada de interés encuentran en los contenidos escolares (Moreno, 2006).

La cotidianeidad en la que viven los niños es tierra fértil para el cultivo de los procesos de autoorganización del aprendizaje que se fortalecen gracias a que perciben, de los adultos, la confianza que los incentiva o el desinterés que los desmotiva. Si un niño se siente libre bajo la mirada y el cuidado del adulto generará más mecanismos de confianza y autonomía en su desarrollo (Erikson, 2000); en caso contrario, se corre el riesgo de generar, con la misma fuerza, mecanismos de defensa donde la inseguridad, el temor y la culpa desestructurarán los andamios de la propensión a aprender dando paso a la latencia cognitiva (Erikson, 2000).

El proceso autoorganizativo se estanca cuando se somete a la hegemonía de la escuela, pero curiosamente, fuera de ella, las relaciones que el niño establece libremente le permiten equilibrar el proceso. Todo ello dependerá, también, de la familia y de la comunidad donde el niño vive y de los valores a los que se aspira (Calvo, 2012; Moreno y Calvo, 2010). Lo señalado, más el proceso y producto de nuestras investigaciones, nos permiten diferenciar categóricamente entre educación y escolarización:

La educación ocurre a lo largo de la vida y no se limita a la escuela. Cuando ocurre en ambientes escolarizados se le llama 'educación formal' o 'educación no formal'. Cuando sucede fuera de la escuela se le denomina educación informal y corresponde a la socialización o enculturación. Estas distinciones son erróneas e inducen a serios errores sobre las características y potencialidades de la educación. La educación formal es cartesiana en su fundamento y orientación, al igual que la educación no formal, que trata de actividades de capacitación y animación socio cultural, breves, dinámicas, flexibles y certificables. Sus participantes, a diferencia de la escuela, tienen orígenes, edades y actividades diferentes que se reúnen por objetivos específicos y temporales, quienes se separan, una vez finalizada la capacitación. La educación informal representa los procesos educativos que tienen lugar en la calle, el café o el patio escolar (Calvo, 2005: 15).

La educación es entendida, entonces, como un proceso de creación de relaciones posibles, mientras que la escolarización se definiría como el proceso de repetición de relaciones preestablecidas por otros.

\subsection{PROPENSIÓN A APRENDER Y MODIFICABILIDAD COGNITIVA}

Todo ser humano propende a aprender desde el momento en que comienza su relación con el medio circundante, independientemente de las capacidades que manifieste. Nace dotado de infinitas posibilidades de desarrollo a la espera de ser actualizadas. Algunos son capaces de nutrirse por sí solos de las experiencias del medio, otros necesitan de la guía de un mediador que las seleccione y organice. Las capacidades están alertas ante las experiencias de aprendizaje que las pongan en juego (Feuerstein, 1991; Feuerstein et al., 1988). Sin embargo, a la par de la potencialidad de desarrollo convive la capacidad para inhibirla, sea porque las funciones cognitivas son deficientes o porque no hay motivación para activarlas. Esto deteriora la propensión a aprender y pavimenta el camino al fracaso en la escuela, donde los alumnos terminan atribuyéndose la responsabilidad del fracaso (López de Maturana, 2010).

Ahora bien, dado que los niños y niñas no crecen en el vacío socio cultural, consideramos que el medio puede potenciar o inhibir su desarrollo cognitivo. Entendemos que el medio lo constituyen los ambientes socioculturales donde el niño vive sus experiencias cotidianas, 
las que contribuirán, en mayor o menor medida, al aprovechamiento de los aprendizajes. Si los ambientes son activo modificantes hay mayor posibilidad de actualizar el desarrollo y viceversa. Sin embargo, un niño que en su hogar no cuenta con un ambiente adecuado puede modificarse cognitivamente en la escuela, siempre que se den las condiciones para hacerlo. Es fácil suponer qué les espera a los niños que no han sido mediados ni en el hogar ni en la escuela.

La modificabilidad cognitiva, entendida como un cambio estructural en los patrones de desarrollo cognitivo (Feuerstein, 1991), sucede porque el ser humano es un sistema abierto a los cambios estructurales y porque el sujeto posee gran plasticidad y capacidad para adaptarse a nuevas situaciones. Dependerá de la calidad de las relaciones entre las personas más cercanas, o sea, la familia, compañeros, amigos y profesores.

Los niños que son mediados intencionalmente tienen mayores probabilidades de modificarse cognitivamente y aprovechar su propensión a aprender, en caso contrario, cuando no hay mediación intencional quedan expuestos, arbitrariamente, a los estímulos del medio y pueden correr el riesgo de privarse de los aprendizajes de su propia cultura.

Investigar a los niños y niñas desde los cuatro a los siete años nos abre una enorme posibilidad de comprender cómo piensan, comparan, relacionan, categorizan, hipotetizan, proyectan relaciones virtuales, entre otras funciones cognitivas responsables del pensamiento inteligente (Feuerstein, 1991).

\subsection{AMBIENTES PROPICIOS PARA EL APRENDIZAJE}

Partimos de la base de que los niños son geniales y que construyen de manera genuina sus aprendizajes, exploran, invierten energía y disfrutan por sus logros, pero pierden la genialidad debido a la verticalidad de los procesos escolares que fragmentan su saber (Román $\&$ Herrera, 2009). La escuela es un lugar donde la sociedad se vertebra y donde el multivariado ambiente que allí se genera sienta las bases de la realidad contextual, social y cultural.

Los ambientes no tienen que ver con recursos materiales, infraestructura o disposiciones curriculares, sino con las interrelaciones que allí se generan. Los definiremos, de acuerdo a Feuerstein (1991), como activos modificantes y pasivos aceptantes. El primero facilita el desarrollo fluido de las capacidades de los niños gracias a un mediador intencional y significativo para lograr la reciprocidad en los aprendizajes. El segundo, entorpece lo anteriormente señalado.

Un ambiente activo modificante promueve la propensión a aprender y la construcción y ejercicio del pensamiento crítico porque los niños ponen en juego su propensión a aprender. Es un entorno enriquecido que estimula la plasticidad cerebral, indispensable para la construcción del conocimiento, donde la mirada epistemológica dota de sentido al proceso educativo e incide en la construcción cotidiana de los conceptos (Marina, 2011).

En un ambiente pasivo aceptante no importa lo que cada uno conoce sino el producto solicitado, tampoco se consideran las preguntas ni las "salidas geniales". No se enseña el significado de la actividad más allá de las necesidades inmediatas o el cumplimiento del requisito de evaluación. Si los niños muestran alguna dificultad en sus aprendizajes, esto se acepta pasivamente, bajando, sin previa mediación, los niveles de complejidad y de abstracción de las tareas. De esa manera, los niños aprenden a quedar relegados de los aprendizajes significativos y trascendentes y, peor aún, se auto-convencen de su propia incapacidad. Aprenden que no pueden aprender (Calvo, 2012). 
Uno de los grandes riesgos de este tipo de ambientes es la restricción del desarrollo de las funciones cognitivas responsables del pensamiento inteligente, puesto que la deficiencia en las funciones cognitivas de un sujeto interviene en la calidad de la recepción, elaboración y entrega de la información, lo que finalmente redunda en la construcción de los aprendizajes y en la optimización de los procesos de desarrollo. Si la deficiencia cognitiva se instala en las aulas los niños, ellos aceptarán tácitamente lo que se les imponga, disminuirán su índice de modificabilidad, anularán su curiosidad natural y aprenderán a fragmentar el mundo y la relación natural entre las cosas y los hechos (López de Maturana, 2010).

De acuerdo a lo precedente, entre otros objetivos dela investigación nos propusimos conocer y describir el tipo de ambientes que propicia la propensión a aprender de los niños.

\section{METODOLOGÍA}

La investigación realizada es un estudio fenomenológico situado en un paradigma interpretativo, razón por la cual la metodología es cualitativa, cuyo carácter es descriptivo, relacional e interpretativo (Canales, 2006; Ruiz, 2003; Sandín, 2003). Las preguntas que han direccionado el proceso investigativo nos han permitido identificar y comprender cualidades y características más que registrar cantidades y frecuencias de aparición de un determinado fenómeno: ${ }^{3}$

- ¿Cómo los niños y niñas explican lo que piensan y hacen?

- ¿Cómo se dan cuenta, a través de sus propias acciones, de sus propios procesos de razonamiento?

- ¿Necesita que le expliquen de diferentes maneras una instrucción o la capta inmediatamente?

- ¿Qué caracteriza la transferencia de lo aprendido a nuevas situaciones de aprendizaje?

- ¿De qué modo los ambientes activo modificantes influyen en la generación de preguntas y respuestas de los niños?

El diseño metodológico contempla la indagación, en diversos niveles y dominios fenomenológicos, de la cognición y aprendizaje infantil donde se caracterizaron los contextos relacionales en que tienen lugar los aprendizajes, los patrones distintivos de la cognición infantil, la diversidad y procesos de configuración de distinciones entre los niños, la emergencia y constricción de la experiencia infantil en la educación formal (grados de libertad y coacción) y la capacidad profesional docente para observar, interpretar y potenciar las diversas manifestaciones de la cognición infantil en contextos educativos formales.

Nos interesan particularmente los niños y las niñas de aquellos sectores considerados más vulnerables de la sociedad chilena y que viven en las ciudades La Serena, Coquimbo y Valdivia. Esta es una condición importante por cuanto queremos investigar a qué se debe la capacidad extraordinaria de los niños para preguntar y responder de modo asombroso; y esa muestra, sin aspiración de ser generalizable, responde a nuestros propósitos. Sin embargo, podría suceder una generalización naturalista (Stake, 2010) o generalización situada (Simons, 2011) donde otros niños se reconocieran con las características detectadas en nuestra muestra si es que presentan rasgos similares.

Insistimos en que estas preguntas han sido, sólo, orientadoras de todo el proceso investigativo. 
Durante cada año de investigación, en términos generales, realizamos el mismo trabajo de campo basado en los registros de observación etnográfica, pues el tema no variaba. Los que cambiaban eran los niños, que a medida que crecían tenían nuevas experiencias, valoraciones, preferencias y juegos.

Observamos diariamente durante las horas lectivas de su educación formal. La intención, como es propio de esta técnica de recogida de datos, fue pasar el mayor tiempo posible con los sujetos que participaron de nuestro estudio. Registramos sus interacciones, conversaciones, movimientos, gestos, actitudes, etc., a través de notas de campo construidas en el mismo momento en el que se estaban produciendo los fenómenos observados (Angrosino, 2012).

Una vez transcritos, los datos producidos se sometieron a análisis de contenido apoyado en el programa computacional Atlas ti 7.0. Los procedimientos de análisis, en general, se ciñeron a las propuestas de la Grounded Theory (Gibbs, 2012; Glaser \& Strauss, 1967; Simons, 2011; Strauss \& Corbin, 2002). Se realizó codificación abierta con categorías emergentes; codificación axial en torno a la agrupación de las categorías emergentes en familias de categorías; y codificación selectiva mediante un trabajo interpretativo que condujo a la elaboración de un modelo interpretativo.

\section{RESULTADOS}

Durante los cuatro años de investigación hemos podido constatar, con desazón, que la extraordinaria capacidad de los niños para preguntar y razonar se va perdiendo paulatinamente por las "llamadas de atención" y la indiferencia de sus profesores ante las ocurrencias de los niños.

Después de analizar los registros etnográficos de observación en las escuelas, podemos señalar que los ambientes no propician el aprendizaje significativo, muy por el contrario, lo inhiben. En el primer y segundo año se observó un tipo de ambiente homogéneo, estructurado y parcelado, con una programación rígida que limita e inhibe las experiencias de éxito, donde hay ausencia de diferenciación psicológica, porque predomina la uniformidad.

En el tercer y cuarto año se observó un ambiente que definitivamente asumió las características de un ambiente pasivo aceptante, donde se restringe la creatividad, el ingenio, la reflexión, la entretención y el sentimiento de ser competente. Hay invisibilidad de la complejidad infantil porque se refuerza la percepción episódica de la realidad, no se aprovechan los errores, hay poca intencionalidad y significado, las experiencias son muy guiadas y lineales y las habilidades lingüísticas son poco aprovechadas, ya que se restringe la posibilidad de diálogo y de construcción de aprendizajes.

Un ejemplo donde se aprecia la poca intencionalidad de la educadora para desarrollar las habilidades lingüísticas y el poco significado de la experiencia es el siguiente:

Educadora: ¿Qué pasa en el invierno?

Niña: ¡hace frío!

Niño: ¿Tiene mucho frío tía?

Educadora: ¿Qué más pasa en el invierno?

Niña: En invierno uno se pone gorro, bufanda

Niño: ¡Y botas! 
Estudios Pedagógicos XLI, N Especial: 169-180, 2015

AMBIENTES EDUCATIVOS ESCOLARES: UNA INVESTIGACIÓN SOBRE LA PROPENSIÓN A APRENDER EN JARDINES INFANTILES CHILENOS

\author{
Educadora: ¿Qué se comerá en invierno? \\ Niño: ¡Naranjas! \\ Otro niño: ¡Mandarinas! \\ Educadora: Lo que se come harto en invierno son los frutos secos, los higos secos, por ejemplo \\ ¿han comido higos secos? \\ Niños: ¡Nooooo! (Registro de Campo)
}

Se observa una relación pedagógica donde pareciera que se está educando aunque lo que se hace realmente es responder a los niños con una mera formalidad. El ambiente educativo formal se limita a cumplir con lo planificado sin sacar provecho a las genuinas respuestas de los niños. Parece ser que la educadora espera la respuesta planificada y, entonces, no escucha. Aprovechar que los niños responden otra cosa podría haber desviado el proceso hacia una variedad de conceptos, sinónimos, relaciones o ejemplos. Tampoco media para ayudar a los alumnos a canalizar su impulsividad, a precisar la información, a explorar sistemáticamente la situación de aprendizaje, a seleccionar los datos relevantes o a proyectar relaciones virtuales.

Por ejemplo, cuando la niña responde “¡hace frío!” ante la pregunta de la educadora: “¿Qué pasa en invierno?”, esta podría haber mediado preguntando ¿Por qué hace frío?, ¿Qué haces cuando tienes frío?, ¿Cómo sabes que hace frío?, sin embargo, la respuesta de la niña es ignorada como también lo es la pregunta de un niño que le dice: "¿Tienes mucho frío tía?” La educadora se limita a seguir con su pregunta: “¿Qué más pasa en el invierno”? (Registro de campo).

De la misma manera, en el resto de la sesión la educadora continúa ignorando las respuestas de los niños/as. Otra de las niñas dice: “¡En invierno uno se pone gorro, bufanda!” y el niño agrega: “¡Y botas!” (Registro de campo). La educadora podría mediar preguntando, por ejemplo ¿Por qué hay que ponerse esas prendas?, ¿Conocen algún lugar donde hace más frío?, ¿Qué pasa en el cuerpo cuando hace frío?, ¿Y cuando hace calor? De esa manera, la educadora los conduciría a plantearse hipótesis, proyectar relaciones virtuales, hacer comparaciones, identificar datos relevantes, planificar la conducta, etc. Todas esas son funciones cognitivas que actualizan el potencial de pensamiento (Feuerstein, 1991).

Lo mismo sucede cuando ella pregunta: “QQué se comerá en invierno?” y los niños responden: “¡Naranjas!, ¡Mandarinas!” (Registro de campo). En este ejemplo nuevamente se pierde la posibilidad de mediar la experiencia, puesto que en vez de aseverar: "Lo que se come harto en invierno son los frutos secos" (Registro de campo), podría haber aprovechado la respuesta de los niños/as preguntando: ¿Cuál es la diferencia entre una naranja y una mandarina? y desde allí seguir mediando de manera significativa para ampliar el espectro de comprensión de los niños/as. Incluso podría provocar algún conflicto cognitivo preguntando: ¿Qué le falta a una mandarina para ser un higo? Este tipo de preguntas que pueden catalogarse como absurdas, provocan un desequilibrio en la mente de los niños, muy favorable para la adquisición de nuevos aprendizajes. Sin embargo, en los ejemplos observados se puede apreciar que, antes de entrar en la escuela, se promueve la repetición, la respuesta estereotipada y la falta de reconocimiento a las capacidades y creatividad de los niños en detrimento del sentido y de la trascendencia de lo aprendido.

El análisis realizado nos permite señalar que los niños no tienen dificultades para explicar verbalmente sus acciones y para manifestar de diversas maneras sus necesidades, del mismo modo que se observan evidentes procesos de razonamiento cuando realizan sus 
tareas; piden explicaciones y transfieren hacia nuevas situaciones. Esas manifestaciones cognitivas se observan en el diario acontecer cuando juegan y se relacionan de manera espontánea, sin embargo, cuando interviene la educadora, esta restringe el ambiente creativo, no obstante lo que se espera es que canalice la gran potencialidad y energía de los niños.

La indiferencia ante las situaciones y experiencias significativas son recurrentes:

Educadora: Miren, aquí están las vocales manuscritas: la a, e, i, o, y la u.

Niña: ¡la vaca eres tú!

Educadora: ¿Ven? Acá están las vocales

Niña: a, e, i, o, u, la vaca eres tú

Educadora: ¡Ya! necesito a los ayudantes para que retiren los estuches y repartan los cartones de bingo...

Niño: ¡Tía, yo en el recreo voy a comprarme algo!

Educadora (se dirige al grupo, no mira al niño): ¡A ver, los estudiantes que repartan los estuches! Continúa repartiendo materiales... (Registro de campo)

La niña del ejemplo hace una rima que podría haber sido aprovechada por la educadora de la misma manera que podría haber mantenido la conversación con el niño, sin embargo, hay indiferencia ante esas manifestaciones infantiles. Este es un ambiente pasivo aceptante generado por la educadora que restringe el sentido de competencia y refuerza la percepción episódica de la realidad. No obstante aquello, los niños aprenden porque la propensión a aprender aún está intacta. El riesgo es inminente a medida que avanzan en los años escolares y se espera de ellos que funcionen de acuerdo al sistema.

Hay que admitir que lo normal en educación, es que la cosa no funcione: que el otro se resista, se esconda o se rebele. Lo normal es que la persona que se construye frente a nosotros no se deje llevar, o incluso se nos oponga, a veces, simplemente, para recordarnos que no es un objeto en construcción sino un sujeto que se construye (Meirieu, 2001: 73).

Por lo tanto, las características que hemos podido identificar en relación a los ambientes pasivos aceptantes están relacionadas con:

- Bajísimas expectativas de aprendizaje.

- Excesivo control por parte de los educadores del acontecer que ocurre en la sala de clases.

- Constantes retos a los niños.

- Participación artificial o pseudo participación: invitación a participar (tomar decisiones) en aquellos aspectos del quehacer educativo que no son del verdadero interés de los niños.

A pesar de la descripción minuciosa realizada de estos ambientes que no ayudan a profundizar en la enriquecedora propensión al aprendizaje, sí hemos podido apreciar algunos aspectos emergentes que nos aporta alguna esperanza en relación al aprendizaje de estos niños y niñas. A partir del currículum oculto manifestado en nuestra observaciones, concretamente a partir de la informalidad propia que emerge en cualquier contexto educativo formal (no todo lo que ocurre en la acción educativa puede ser controlado), hay algunos aspectos que consideramos pudieran ayudarnos a comprender los ambientes donde la propensión a aprender puede emerger. A saber: 
- Libertad en la organización: no puede ser confundida con el libertinaje en donde cada uno puede hacer lo que le plazca y en donde no existen reglas que cumplir. Las reglas y los roles existen, pero estos son modificables en el tiempo y el espacio por los mismos actores ejecutantes. La diferencia radica en la autoorganización propia de los niños.

- Confianza: se da entre los propios actores implicados en una acción y el resto de la comunidad, que directa y/o indirectamente están vinculados. Se materializa en una conducta de aceptación de los otros, lo cual no exime de disputas, peleas o actitudes agresivas, sino que estas conductas son secundarias a una lógica mayor basada en la inclusión de los otros.

- Incentivación de la curiosidad/Pedagogía de la pregunta: a mayor curiosidad por aquello en lo que se está participando, mayor posibilidad de que el proceso de aprendizaje sea genuino. La confianza mostrada anteriormente origina y permite la pregunta basada en un verdadero interés por conocer, por saber y por poder seguir haciendo, de la mejor manera posible, algo en lo que se está implicado.

- Aprendizaje práctico: es determinante en los ambientes que propician la propensión a aprender de los niños. Está centrado en el saber hacer, concreto y operacionalizado en la cotidianeidad, lo que no excluye las preguntas que buscan saciar los por qué, para qué, etc.; pero el centro está en el hacer cotidiano.

\section{DISCUSIÓN Y CONCLUSIONES}

Uno de los desafíos más difíciles de la escuela es el cambio en el pensamiento del profesorado ante la complejidad de los procesos educativos (Jiménez \& Feliciano, 2006). Cuando esta no se entiende, se la complica terminando por superficializar el proceso de enseñanza y el de aprendizaje (Calvo, 2012). Cuando eso sucede se lo reduce a una linealidad racional que deja fuera la historia, los sentimientos, la emocionalidad y otros factores intervinientes en la integralidad de los niños.

Pareciera ser que los contextos inestables inquietan al profesorado que no sabe qué hacer con la incertidumbre, aunque esta forma parte del proceso de desarrollo de los niños y del sentido común en relación con la experiencia directa con el medio. Se busca la certeza predefiniendo su mundo (Hargreaves, 2005).

Si los dejáramos hacer en un marco de libertad responsablemente intencionada, quizá cuántos aprendizajes descubriríamos sobre las maneras de conocer. Si eso sucediera sería crucial para la formación inicial de profesores, pues podríamos mostrarles que los niños son geniales, que no deben forzarlos para aprender y que no siempre deben hacerlo de acuerdo a la planificación adulta.

Por lo general, nos encontramos con niños cuyo repertorio cognitivo es mal utilizado solo porque no encuentran las estrategias o las maneras para manifestarlo. Por eso, es necesario encontrar el índice de modificabilidad que a veces está oculto, pero que existe en cada niño, y proporcionarle las condiciones internas y externas para que lo manifieste (López de Maturana, 2010).

La mediación se presenta como la alternativa viable para construir conjuntamente los aprendizajes y mejorar la calidad de las interrelaciones porque es intencional para buscar la reciprocidad, significativa para dar sentido a las cosas y a los hechos y trascendente para pasar más allá del aquí y del ahora.

El aprendizaje mediado permite que los niños aprendan a beneficiarse por sí solos de los estímulos provenientes del medio porque es un gran impulsor de la modificabilidad 
cognitiva. También promueve el desarrollo de las habilidades sociales para interactuar con sus pares y con los adultos con quienes conviven, solucionar problemas y tomar decisiones acertadas. Se desarrolla el locus de control interno para ser capaces de reconocer sus propias emociones y las de los demás en un marco de respeto y solidaridad.

Los niños no son conscientes del desarrollo de sus funciones cognitivas y de cómo pueden potenciarlas, lo que puede llevarlos a la desmotivación ante una tarea que les presenta dificultad o si sus ocurrencias no reciben retroalimentación de los adultos. Por lo tanto, la mediación es fundamental para mantener el entusiasmo en el aprendizaje y la construcción conjunta de los saberes. La promoción de la pedagogía debería tenerlo en cuenta para evitar la exclusión de la diversidad.

El desarrollo de la estructura cognitiva de un ser humano se concibe como el producto de dos modalidades de interacción entre el organismo y su medio. La primera considera que la exposición directa del organismo al estímulo produce cambios en el niño, pero carentes de intencionalidad, significado y trascendencia. La segunda considera la transformación del estímulo a través de un mediador que selecciona y organiza los estímulos cuando el niño tiene dificultad para hacerlo por sí solo. Estos se encuentran en la familia, el jardín infantil, la escuela, el barrio, etc., (Feuerstein, 1991).

Lo anterior adquiere mayor relevancia cuando el niño pasa desde el nivel de educación parvularia a la escuela, donde la falta de articulación entre ambos provoca un vacío cognitivo y emocional que termina convenciendo al niño que ya no puede preguntar (Calvo, 2011). Por lo tanto, se necesita un ambiente que transforme lo pasivo aceptante en activo modificante y que proporcione una sólida base conceptual desde el inicio de la escolaridad.

Si el educador, con el pretexto de no perder el tiempo, deja de contar historias a los niños, siempre habrá comerciantes dispuestos a hacerlo y la astucia comercial recurrirá a todas sus mañas, combinándolas para no perder la venta. Los chicos acuden como moscas a esa bazofia, mientras que los educadores torpes o ineptos desatienden una de las necesidades iniciales de los niños de nuestro tiempo.... Treinta niños te escuchan ¿qué esperan de ti? Que tu voz, que lo que vas a contar se relacione con su experiencia... (Deligny Cit. en Meirieu, 2010: 176).

En la presentación del proyecto señalamos que el paso del Jardín infantil al Primero Básico constituye un quiebre en la vida del niño que debe ser considerado por los educadores; sin embargo, al parecer la tensión entre ambas modalidades se está resolviendo a favor de la paulatina escolarización del Jardín Infantil (Calvo, 2011).

Ahora, tras concluir nuestro trabajo investigativo, podemos señalar que la distancia es cada vez más corta y que, si bien la distribución del espacio es diferente, las prácticas en los jardines infantiles son tan escolarizantes como las de la escuela. Nuestros registros de observación así lo evidencian.

El riesgo es que los niños acepten tácitamente lo que se les imponga, vayan escolarizándose desde los primeros años y repitiendo mecánicamente los contenidos, lo que implica una baja en su índice de modificabilidad cognitiva. Este índice es la capacidad de cada niño para utilizar sus funciones cognitivas de manera óptima, pero para eso necesita mediación. De la mediación de los adultos depende que el sistema holístico de pensamiento infantil evolucione a etapas más complejas de desarrollo. 


\section{REFERENCIAS BIBIOGRÁFICAS}

Angrosino, M. (2012). Etnografía y Observación Participante en Investigación Cualitativa. Madrid: Editorial Morata.

Calvo, C. (2004). Educació y propensió a aprendre i a ensenyar. En P. Aparicio (Ed.), Ètica, complexitat i formació de persones adultes en una societat planetària (pp. 71-93). Valencia: Edicions del Crec.

Calvo, C. (2005). Complejidad, Caos y Educación. En A. Arellano (Coord.). La educación en tiempos débiles e inciertos (pp. 115-136). Barcelona: Antrophos.

Calvo, C. (2011). Asombros educativos infantiles y propensión a aprender. Proyecto de Investigación Fondecyt N ${ }^{\circ} 1110577$. Santiago de Chile: Fondo Nacional de Investigación Científica y Tecnológica.

Calvo, C. (2012). Del mapa escolar al territorio educativo. Disoñando la escuela desde la educación (4a ed.). La Serena: Editorial Universidad de La Serena.

Canales, M. (2006). Metodologías de Investigación Social. Introducción a los oficios. Santiago de Chile: LOM Ediciones.

Erikson, E. (2000). El ciclo vital completado. Barcelona: Editorial Paidós.

Feuerstein, R. (1991). Mediated Learning Experience: A theoretical Review. En R. Feuerstein, P. Klein, A. Tannenbaum (Coords.), Mediated Learning Experiencie: theoretical, psychosocial and learning implications (pp. 3-51). London: Freund Publishing House.

Feuerstein, R., Rand, Y., \& Rynders, J. (1988). Don't Accept Me As I Am: Helping 'Retarted' People to Excel. New York: Plenum Press. Morata.

Gibbs, G. (2012). El análisis de datos cualitativos en investigación cualitativa. Madrid: Editorial

Glaser, B., \& Strauss, A. (1967). The discovery of grounded theory. Strategies for qualitative research. Chicago: Aldine Publishing Company.

Hargreaves, A. (2005). Profesorado, Cultura y Postmodernidad. Madrid: Editorial Morata.

Jiménez, A., \& Feliciano, L. (2006). Pensar el pensamiento del profesorado. Revista Española de Pedagogía, LXIV(233), 105-122.

López de Maturana, S. (2010). Maestros en el territorio. La Serena: Editorial Universidad de La Serena.

Marina, J. A. (2011). El cerebro infantil: la gran oportunidad. Madrid: Editorial Ariel.

Meirieu, P. (2001). Frankenstein educador. Madrid: Laertes.

Meirieu, P. (2010). Una llamada de atención. Cartas a los mayores sobre los niños de hoy. Barcelona: Ariel.

Moreno, A. (2006). Teoría del caos y educación informal. Huelva: Editorial Hergué.

Moreno, A., \& Calvo, C. (2010). Etnoeducación, Educación Física y Escuela: transitando desde la educación informal a la escuela autoorganizada. Revista Ágora para la Educación Física y el Deporte, 12(2), 131-150.

Román, E., \& Herrera, J. (2009). Enseñar y aprender en la Sociedad del Conocimiento: el trabajo independiente y la labor del tutor una alternativa para su concreción. Cuadernos de Educación y Desarrollo, 1(1). Recuperado desde http://www.eumed.net/rev/ced/01/labor_del_tutor.htm

Ruiz, J. (2003). Metodología de la investigación cualitativa. Bilbao: Editorial Universidad de Deusto.

Sandín, M. (2003). Investigación Cualitativa en Educación. Fundamentos y Tradiciones. Madrid: Editorial McGraw Hill.

Simons, H. (2011). El estudio de caso: teoría y práctica. Madrid: Editorial Morata.

Stake, R. (2010). Investigación con estudio de casos. Madrid: Editorial Morata.

Strauss, A., \& Corbin, J. (2002). Bases de la investigación cualitativa. Técnicas y procedimientos para desarrollar la teoría fundamentada. Medellín: Editorial Universidad de Antioquia.

Wood, D., Bruner, J., \& Ross, G. (1976). The role of tutoring in problem solving. Journal of Child Psychology and Psychiatry, 17(2), 89-100. 